\title{
Comparison of videolaryngoscopy and direct laryngoscopy by less experienced paramedics during out-of-hospital cardiopulmonary resuscitation. An observational prospective study.
}

Joachim Risse ( $\nabla$ joachim.risse@uk-essen.de)

Universitatsklinikum Essen https://orcid.org/0000-0002-1671-8506

Christian Volberg

Philipps-Universitat Marburg

Thomas Kratz

Philipps-Universitat Marburg

Birgit Plöger

Philipps-Universitat Marburg

Andreas Jerrentrup

Philipps-Universitat Marburg

\section{Dirk Pabst}

Uniklinik Essen

Clemens Kill

Uniklinik Essen

\section{Research Article}

Keywords: Cardiopulmonary resuscitation, Endotracheal intubation, Paramedics, Out-of-hospital cardiac arrest, Videolaryngoscopy

Posted Date: November 15th, 2019

DOI: https://doi.org/10.21203/rs.2.16606/v1

License: (c) (1) This work is licensed under a Creative Commons Attribution 4.0 International License. Read Full License

Version of Record: A version of this preprint was published at BMC Emergency Medicine on March 23rd, 2020. See the published version at https://doi.org/10.1186/s12873-020-00316-z. 


\section{Abstract}

Introduction: Videolaryngoscopy (VL) has become a popular method of intubation (ETI). Although VL may facilitate ETI in less-experienced rescuers there are limited data available concerning ETI performed by paramedics during CPR. The goal was to show the benefit of VL during CPR performed by nonphysicians in a single emergency department in Germany. We investigated in an observational prospective study the superiority of VL for less-experienced paramedics during CPR compared with direct laryngoscopy (DL).

Methods: We instructed and equipped paramedics from four ambulances in endotracheal intubation (ETI) with GlideScope ${ }^{\circledR}(G V L)$. The primary endpoint was good visibility of the glottis (Cormack-Lehane grading $1 / 2)$, and the secondary endpoint was successful intubation.

Results: In total $n=97$ patients were included, $n=69$ using DL (with $n=85$ intubation attempts) and $n=$ 28 using VL (with $n=37$ intubation attempts). Videolaryngoscopy resulted in a significantly improved visualization of the larynx compared with $\mathrm{DL}$. In the group using GVL, 82\% rated visualization of the glottis as $C L 1 \& 2$ versus $55 \%$ in the $D L$ group $(p=0.02)$. Despite better visualization of the larynx, there was no statistically significant difference in successful ETI between GVL and DL (GVL 75\% vs. DL 68.1\%, $p=0.63)$.

Conclusions: When used by paramedics during CPR, GVL led to better visibility of the glottis but did not increase the number of successful initial intubation attempts. Therefore, we conclude that education in VL should also focus on insertion of the endotracheal tube, considering the different procedures of GVL.

\section{Introduction}

Maintaining an open airway is one of the most important procedures in emergency care during advanced life support (ALS) resuscitation and is essential for adequate ventilation of the patient. Emergency medical services (EMS) in Germany is designed as a two-tiered system including a physician-staffed EMS unit in all life-threatening cases. As soon as the EMS physician is on scene, procedures such as endotracheal Intubation (ETI) must be performed by the physician. Due to the higher availability of paramedic-staffed ambulances, in many cases the paramedics are on scene before the arrival of the EMS physician and are trained in full ALS.

We investigated the benefit of using videolaryngoscopy $(\mathrm{VL})$ instead of direct laryngoscopy (DL) by paramedics in out-of-hospital cardiac arrest before arrival of the EMS physician on scene in a semi-rural county in Germany. An early and safe ETI is associated with an increased likelihood of return of spontaneous circulation (ROSC), an improved neurological outcome, and better patient survival [1, 2]. Adequate ventilation, improved oxygenation, and avoidance of aspiration are important factors concerning the rate of ROSC as well as the neurological outcome of a patient undergoing CPR $[3,4]$. Patients undergoing CPR are associated with a difficult airway [5]. The American Heart Association AHA and the European Resuscitation Council ERC recommend ETI as the gold standard for securing the 
airway in patients undergoing CPR [6,7]. ETI offers protection against aspiration [8]. In the hospital under ideal conditions during elective ETI, a difficult airway occurs in $3.2 \%$ of cases, difficult laryngoscopy in $5.8 \%$, and suboptimal glottic view in $9 \%[9,10,11]$. The American Heart Association (AHA) and the European Resuscitation Council (ERC) recommended ETI as the gold standard for securing the airway in patients undergoing CPR, in case of less experienced providers they recommended mask ventilation or supraglottic devices in order to not interrupt chest compressions. But there are less valid data for difficult ETI in the out of hospital setting.

In the Anglo-American paramedic system, the success rates for prehospital ETI using DL are between $71 \%$ and $75 \%[12,13]$. For German paramedics, ETI during CPR is generally a rare event as the attending physician usually carries out the procedure. Some investigators have shown that untrained users have a $51 \%$ rate of successful intubation with DL (Maclntosh) [14]. In contrast, with VL inexperienced users have an especially steep learning curve and a significantly higher success rate $[14,15]$. Videolaryngoscopy is superior to $D L$ when the first attempt at intubation has failed and is associated with a reduction in esophageal intubations $[16,17]$. Moreover, data exist showing that the success rate of intubation by inexperienced physicians during CPR is significantly higher with $\operatorname{VL}$ than with $D L[17,18,19]$. The advantage of using VL for endobronchial intubation during CPR might result in reduced interruption of chest compressions $[20,21,22]$. Several studies have shown that in pre-hospital settings there is an alarmingly high rate of failed ETI, especially when performed by non-physicians $[23,24,25]$. The goal of our study is to show the superiority of VL for less-experienced paramedics during CPR compared with DL.

\section{Methods}

\section{Study Design and Time Period}

With approval by the institutional ethics committee (EudraCT number 2015-000919-41, retrospectively registered), we designed a prospective observational study comparing DL and GVL by paramedics in outof-hospital cardiac arrest (OHCA) without an EMS physician on scene. We performed our investigation under actual field conditions over a period of 4 years to include a sufficient number of cases. Intubation should be performed either with VL (GlideScope ${ }^{\circledR}$ Ranger, GVL) or the standard DL (Maclntosh) depending on the availability of VL at time of CPR. Therefore four videolaryngoscopes rotated on the ambulances over the period of 4 years. These four videolaryngoscopes were alternating on 32 ambulances of the emergency medical service during the investigation period.

\section{Study Setting and Population}

The inclusion criteria were patients in non-traumatic cardiac arrest, ongoing basic life support (BLS) with chest compressions and bag-mask-ventilation, and absence of an emergency physician on scene. Intubation should be performed either with VL (GlideScope ${ }^{\circledR}$ Ranger, GVL) or DL (Maclntosh) depending on the availability of VL at time of CPR. Four videolaryngoscopes rotated on the ambulances over a period of 4 years. The Videolaryngoscopes were alternating on 32 ambulances of emergency medical service. 
The exclusion criteria were patients aged less than 12 years, the presence of an emergency physician on scene, and the primary use of a supraglottic airway device by the paramedics.

Paramedics from the ambulance bases received a training course explaining handling of GVL before starting the study. For this purpose, an exercise phantom head was used for intubation training, and the correct handling of the GVL was practiced under medical supervision, ca. 20 intubations (JR, TK, CK). A new standard operating procedure (SOP) "Airway management with videolaryngoscopy (GVL)" was developed before commencing the study and was implemented in the annual paramedic training. The new SOP and an instruction manual were made available to all paramedics via the company's intranet.

An intubation attempt was defined as the use of VL or DL to secure the airway at any time during advanced cardiac life support (ACLS). Our primary endpoint was the visibility of the glottis with a Cormack-Lehane $(\mathrm{CL})$ score of 1 or 2, and the secondary endpoint was the overall ETI success rate during out-of-hospital CPR. The number of required attempts was also recorded. Successful ETI was defined as the successful placement of an endotracheal tube and correct pulmonary ventilation with a positive capnography. A maximum of two attempts were allowed per the internal ALS protocol of the emergency medical service. If ETI failed, paramedics were recommended to use a laryngeal mask to secure the airway. After every ETI performance during CPR by paramedics, the research team sent a self-report questionnaire to the paramedic team to acquire data.

\section{Outcome Measures and Data Analysis}

Non-normally distributed variables were expressed as the median and upper and lower quartiles (Q.25 and Q.75). These data were analyzed by using the Mann-Whitney $U$ test. Data were also presented as percentages with confidence intervals $(\mathrm{Cl})$. Differences in frequency were tested for significance using the chi-square test and for small cell occupations using Fisher's exact test. To test for correlations, the point biserial correlation coefficient (r.pb) was used. The significance level was set to alpha $=0.05$. Data are presented as histograms and box-and-whisker diagrams. All statistical calculations were performed using the statistics packages SPSS (version 22) and BiAS for Windows (version 11.03, Epsilon Verlag, 2016).

\section{Results}

In total 134 case report forms (CRF) from patients after CPR were collected. For various reasons, $37 \mathrm{CRF}$ were excluded from further evaluation (see Figure 1). Finally, the CRF's of 97 patients were included. In total $n=97$ patients were included, $n=69$ using $D L$ (with $n=85$ intubation attempts) and $n=28$ using GVL (with $n=37$ intubation attempts). Paramedics' professional experience in years (7 in DL vs 6.5 in $G V L, p=0.48$ ) and the estimated number of intubations (30 vs $22.5, p=0.14$ ) performed previously by the paramedics were similar in both groups. For the $C L$ grade $1-4$, a statistically significant difference in our data could be shown between the two groups (Mann-Whitney $U$ test: $p=0.002$, two-tailed) (see Figure 2). With GVL a CL grade of 1 was significantly more frequent, with a difference of $27.7 \%$ compared with DL ( $p=0.004$ ) (see Table 1$)$. The proportion of dichotomized CL grades $1 \& 2$ vs $3 \& 4$ were statistically significantly different in the GVL group compared with the DL group. CL grades $1 \& 2$ represent an easier 
ETI in contrast to Grades $3 \& 4$, which represent a more difficult ETI. CL grade $1 \& 2$ was statistically significantly more frequent in the GVL group $(23 / 28,82,2 \%(95 \% \mathrm{Cl} 68 \%-96 \%)$ ) than in the DL group $(38 / 69,55,1 \%(95 \% \mathrm{Cl} 43 \%-67 \%))$. The P-value calculated with the two-tailed Fisher's exact test was 0.02 (see Table 1). Regardless of the method used for an ETI by paramedics, our data showed that the number of unsuccessful ETIs increased with a higher CL grade $(r . p b=0.614, p<0.0001)$ (see Table 2).

The other focus of the investigation (overall ETI success) did not differ significantly between the GVL group $(21 / 28,75 \%$ (95\% Cl 59\%-91\%)) and the DL group $(47 / 69,68.1 \%$ (95\% Cl 57\%-79\%)). The P-value calculated with the two-tailed Fisher's exact test was 0.63 (see Table 3). There was no statistically significant difference between the two groups in the frequency of intubation attempts (1 or 2), ( $p=0.4)$ (see Table 4). Also, the success rate on the first and second attempt was similar in both groups. The success rate on the first attempt was 15/21, 71.4\% (95\% Cl 52\%-90\%) in the GVL group and 40/47, $85.1 \%(95 \% \mathrm{Cl} 75 \%-95 \%)$ in the DL group. The success rate on the second attempt was $6 / 21,28.6 \%$ (95\% $\mathrm{Cl} 10 \%-48 \%)$ in the GVL group versus $7 / 47,14.9 \%(95 \% \mathrm{Cl} 5 \%-25 \%)$ in the DL group. The difference found was not significant $(p=0.2)$ (see Table 5).

\section{Discussion}

Paramedics with limited experience in $\mathrm{DL}$ and videolaryngoscopic intubation might have improved success rates using GVL as a first-line device in emergency airway management with CPR. Our results from the present out of hospital observation study demonstrate better visualization of the larynx with GVL. A higher proportion of CL grade $3 \& 4$ was associated with a difficult to impossible ETI, and ETI with GVL received a statistically significantly improved visualization of the larynx compared with DL. Therefore in terms of visualization of the larynx, our data substantiated the superiority of GVL to DL. Despite better visualization of the larynx with GVL, the overall success rates for intubation on the first and second attempt was not improved compared with DL during cardiopulmonary resuscitation when performed by less experienced paramedics.

Previous investigations showed a significantly higher intubation success rate by inexperienced users during CPR with GVL than with DL; however [18, 21], we were not able to show an increased success rate for ETI when performed by paramedics inexperienced in the procedure, despite repeatedly proven improved visualization of the larynx via GVL.

Lee et al. investigated tracheal intubation during in-hospital cardiopulmonary resuscitation [18]. But results from clinical research cannot simply be transferred to the out of hospital setting. Park et al. investigated tracheal intubation during cardiopulmonary resuscitation by novice physicians [21]. Endotracheal intubation during resuscitation is frequently associated with a difficult airway and shows a success rate on the first attempt with VL, depending on the study, between $73 \%$ and $94 \%$, even for experienced physicians $[18,22]$. Most of the previous studies observing GVL during CPR investigated experienced physicians or were just simulation studies with mannequins $[8,22,26,27]$. The differences to 
our results might be based on user experience (physicians, non-physicians) with the procedure. We suspect a broad range of user experiences across individuals and studies.

Ducharme et al. saw similar relevant results in their investigation of American paramedics over a period of 34 months. The group showed that VL had similar first-attempt success rates and even better laryngoscopic visualization compared with DL. They used the King Vision® videolaryngoscope, whereas our investigation used the GlideScope ${ }^{\circledR}$ Ranger [28]. In addition, our study results showed a trend towards a higher rate of successful ETI on the second attempt with GVL. This might be based on an immediate learning process from the first attempt to the second attempt with VL. A minimal optimization during the second attempt (blood and secretion suction, cleaning the lens, view of the monitor) might be enough in such a situation to successfully intubate with GVL. Nouruzi-Sedeh et al. showed a success rate of more than $90 \%$ on the first attempt in their investigation with personnel untrained in intubation using GVL. In the second attempt, all subjects were successfully intubated with the GlideScope technique [14]. In this context, due to the small number of cases, we could only see a statistically insignificant trend in our data towards a higher rate of successful ETI on the second attempt with GVL during CPR.

During out-of-hospital CPR there are a numerous external influences and stressors on the paramedic team, for example, the unfamiliar environment, lighting etc. Russo et al. postulate that videolaryngoscopes are helpful for emergency intubations, but sufficient experience in dealing with the devices is essential. They also showed the limitations of videolaryngoscopes, e.g. blood, vomit or secretions in front of the lens, as well as bright light producing glare on the screen [28]. Finally, there are numerous internal and external stressors on paramedics when performing an intubation during resuscitation. These stressors might also be responsible for the poor performance observed with both devices.

\section{Limitations}

The first limitation of our study is related to its design. We performed a preliminary observation trial with less experienced paramedics from a single emergency department. For that reason, our study sample was small and unbalanced. For paramedics in Germany, ETI is an extremely rare event, and we performed our investigation under actual field conditions over a period of 4 years to include a sufficient number of cases. In most cases of pre-hospital emergency medicine in Germany, an emergency physician performs intubation. To obtain a larger case number in an adequate investigation time period, several different emergency departments should be included in further investigations. In addition, there is a possibility of reporting bias. Despite anonymization of the questionnaires and optional participation for the paramedics in this investigation, positive results and positive occurrences might be reported more often than negative ones. A further limitation is due to the different levels of training with DL and GVL of the individual paramedics in the single investigated emergency department. Based on the variability of individual intubation experience among paramedics in this single emergency medical service, the results cannot be transferred to other emergency departments. 
Furthermore, we used the GlideScope ${ }^{\circledR}$ Ranger videolaryngoscope in our investigation, while the group of Ducharme et al. for example used the King Vision ${ }^{\circledR}$ videolaryngoscope [29]. The two studies obtained similar results; however, there are currently many different videolaryngoscopes with varying designs and quality available on the market. For these reasons, our study results should not be generalized, and further investigation is needed.

\section{Conclusion}

At present, the rate of successful ETI performed by less experienced paramedics during cardiopulmonary resuscitation is not significantly improved with the use of VL compared with $D L$. This may be due to less experience in handling a videolaryngoscope and infrequent opportunities for German paramedics to perform an intubation in general. It is also possible that $\mathrm{VL}$ is not as intuitive as one might assume. When used by paramedics during CPR, VL led to better visibility of the glottis but did not increase the number of successful initial intubation attempts. In the future, a wide use of VL in the emergency service combined with intensive training for all levels of the rescue personnel could improve performance for endobronchial intubation during CPR with VL.

\section{List Of Abbreviations}

AHA - American Heart Association

ACLS - Advanced cardiac life support

ALS - Advanced life support

CL grading - Cormack-Lehane grading

CPR - Cardiopulmonary resuscitation

CRF - Case report form

CK - Clemens Kill

EMS - Emergency medical services

ERC - European resuscitation council

ETI - Endotracheal intubation

DL - Direct laryngoscopy

GVL - GlideScope ${ }^{\circledR}$ videolaryngoscopy

JR - Joachim Risse 
OHCA-Out-of-hospital Cardiac Arrest

ROSC - Return of spontaneous circulation

SOP - Standard operating procedure

TK - Thomas Kratz

VL - Videolaryngoscopy

\section{Declarations}

\section{Ethics approval and consent to participate}

Ethics approval was granted by the Ethics Committee of Philipps-University Marburg, Baldingerstrasse/Postfach 2360, 35032 Marburg, Germany Az.: 184/10, 10.11.2010.

Consent to participate was not required. This investigation in the context of resuscitation requires no written consent of the resuscitated patients in the context of quality assurance. The "Ordinance on Quality Assurance in Rescue Services" of 27 February 2003 (GVBI. I p. 105), issued based on section 26 (1), (3) and (4) and section 27 (1) of the Hessian Rescue Service Act 1998 of 24 November 1998 (GVBI. I p. 499) explicitly commits the institutions responsible for emergency care within the rescue service to anonymous data collection and evaluation in the context of quality assurance. The inclusion of clinical follow-up data in conjunction with Section 12 (2) no. 8 of the Hessian Hospital Act 2002 of 6 November 2002 (GVBI. I p. 662) is also finally regulated. With reference to this legal basis, the Ethics in Medicine Commission of the Philipps-University of Marburg agreed in 2010 to the investigation.

\section{Consent for publication}

Not applicable

\section{Availability of data and material}

The data that support the findings of this study are available from the corresponding author. The datasets used and analyzed during the current study are available from the corresponding author on reasonable request.

\section{Competing interests}

The authors declare that they have no competing interests.

\section{Funding}

No funding 


\section{Authors' contributions}

$\mathrm{JR}$ and $\mathrm{CV}$ analyzed and interpreted the patient data. JR was the major contributor in writing the manuscript. All authors read and approved the final manuscript.

\section{Acknowledgments}

Comesta (Consulting Methods \& Statistics), Dipl.-Psych. Thomas Ploch for his statistical support.

The authors report no conflicts of interest

\section{References}

1. Benoit JL, Gerecht RB, Steuerwald MT, et al. Endotracheal intubation versus supraglottic airway placement in out-of-hospital cardiac arrest: A meta-analysis. Resuscitation. 2015;93:20-6.

2. Wang HE, Szydlo D, Stouffer JA, et al. Endotracheal intubation versus supraglottic airway insertion in out-of-hospital cardiac arrest. Resuscitation. 2012;83:1061-6.

3. Spindelboeck W, Schindler O, Moser A, et al. Increasing arterial oxygen partial pressure during cardiopulmonary resuscitation is associated with improved rates of hospital admission. Resuscitation. 2013;84:770-5.

4. Yeh ST, Cawley RJ, Aune SE, et al. Oxygen requirement during cardiopulmonary resuscitation (CPR) to effect return of spontaneous circulation. Resuscitation. 2009;80:951-5.

5. Khandelwal N, Galgon RE, Ali M, et al. Cardiac arrest is a predictor of difficult tracheal intubation independent of operator experience in hospitalized patients. BMC Anesthesiol. 2014;14:38.

6. Graesner JT, Bein B. Reanimation- Erweiterte Maßnahmen für Erwachsene (Adult Advanced Life Support). Anästhesiol Intensivmed Notfallmed Schmerzther 2016;51: 188-94.

7. Link MS, Berkow LC, Kudenchuk PJ, et al. Part 7: adult advanced cardiovascular life support: 2015 American Heart Association Guidelines Update for Cardiopulmonary Resuscitation and Emergency Cardiovascular Care. Circulation. 2015;132(November (18 Suppl. 2)):S444-64.

8. Piegeler T, Roessler B, Goliasch G, et al. Evaluation of six different airway devices regarding regurgitation and pulmonary aspiration during cardio-pulmonary resuscitation (CPR) - A human cadaver pilot study. Resuscitation. 2016;102:70-4.

9. Combes X, Le Roux B, Suen P et al. Unanticipated difficult airway in anesthetized patients: prospective validation of a management algorithm. Anesthesiology. 2004;100:1146-50.

10. Shiga T, Wajima Z, Inoue T et al. Predicting difficult intubation in apparently normal patients: a metaanalysis of bedside screening test performance. Anesthesiology. 2005;103:429-37.

11. Thoeni $\mathrm{N}$, Piegeler T, Brueesch $\mathrm{M}$ et al. Incidence of difficult airway situations during prehospital airway management by emergency physicians-A retrospective analysis of 692 consecutive patients. 
Resuscitation. 2015;90:42-5.

12. Myers LA, Gallet CG, Kolb LJ, et al. Determinants of success and failure in prehospital endotracheal intubation. West J Emerg Med. 2016;17:640-7.

13. Voss S, Rhys M, Coates D, et al. How do paramedics manage the airway during out of hospital cardiac arrest? Resuscitation. 2014;85:1662-6.

14. Nouruzi-Sedeh P, Schumann M, Groeben H. Laryngoscopy via Macintosh blade versus GlideScope: success rate and time for endotracheal intubation in untrained medical personnel. Anesthesiology. 2009;110:32-7.

15. Sakles JC, Mosier J, Patanwala AE, et al. Learning curves for direct laryngoscopy and GlideScope ${ }^{\circledR}$ video laryngoscopy in an emergency medicine residency. West J Emerg Med. 2014;15:930-7.

16. Sakles JC, Javedani PP, Chase E, et al. The use of a video laryngoscope by emergency medicine residents is associated with a reduction in esophageal intubations in the emergency department. Acad Emerg Med. 2015;22:700-7.

17. Sakles JC, Mosier JM, Patanwala AE, et al. The C-MAC® video laryngoscope is superior to the direct laryngoscope for the rescue of failed first-attempt intubations in the emergency department. J Emerg Med. 2015;48:280-6.

18. Lee DH, Han M, An JY, et al. Video laryngoscopy versus direct laryngoscopy for tracheal intubation during in-hospital cardiopulmonary resuscitation. Resuscitation. 2015;89:195-9.

19. Myers LA, Gallet CG, Kolb LJ, et al. Determinants of success and failure in prehospital endotracheal intubation. West J Emerg Med. 2016;17:640-7.

20. Park SO, Baek KJ, Hong DY, et al. Feasibility of the video-laryngoscope (GlideScopeß) for endotracheal intubation during uninterrupted chest compressions in actual advanced life support: a clinical observational study in an urban emergency department. Resuscitation. 2013;84:1233-7.

21. Park SO, Kim JW, Na JH, et al. Video laryngoscopy improves the first-attempt success in endotracheal intubation during cardiopulmonary resuscitation among novice physicians. Resuscitation. 2015;89:188-94.

22. Kim JW, Park SO, Lee KR, et al. Video laryngoscopy vs. direct laryngoscopy: Which should be chosen for endotracheal intubation during cardiopulmonary resuscitation? A prospective randomized controlled study of experienced intubators. Resuscitation. 2016;105:196-202.

23. Cobas MA, De la Peña MA, Manning R, et al. Prehospital intubations and mortality: a level 1 trauma center perspective. Anesth Analg. 2009;109:489-93.

24. Timmermann A, Russo SG, Eich C, et al. The out-of-hospital esophageal and endobronchial intubations performed by emergency physicians. Anesth Analg. 2007;104:619-23.

25. Ufberg JW, Bushra JS, Karras DJ, et al. Aspiration of gastric contents: association with prehospital intubation. Am J Emerg Med. 2005;23:379-82.

26. Hossfeld B, Frey K, Doerges V et al. Improvement in glottic visualisation by using the C-MAC PM video laryngoscope as a first-line device for out-of-hospital emergency tracheal intubation: An 
observational study. Eur J Anaesthesiol. 2015;32:425-31.

27. Shin DH, Choi PC, Han SK: Tracheal intubation during chest compressions using Pentax-AWS(®), GlideScope $(\circledR)$, and Macintosh laryngoscope: a randomized crossover trial using a mannequin. Can J Anaesth. 2011;58:733-9.

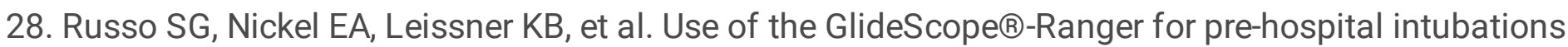
by anaesthesia trained emergency physicians - an observational study. BMC Emerg Med. 2016;16:8.

29. Ducharme S, Kramer B, Gelbart D, et al. A pilot, prospective, randomized trial of video versus direct laryngoscopy for paramedic endotracheal intubation. Resuscitation. 2017;114:121-6.

\section{Figures}

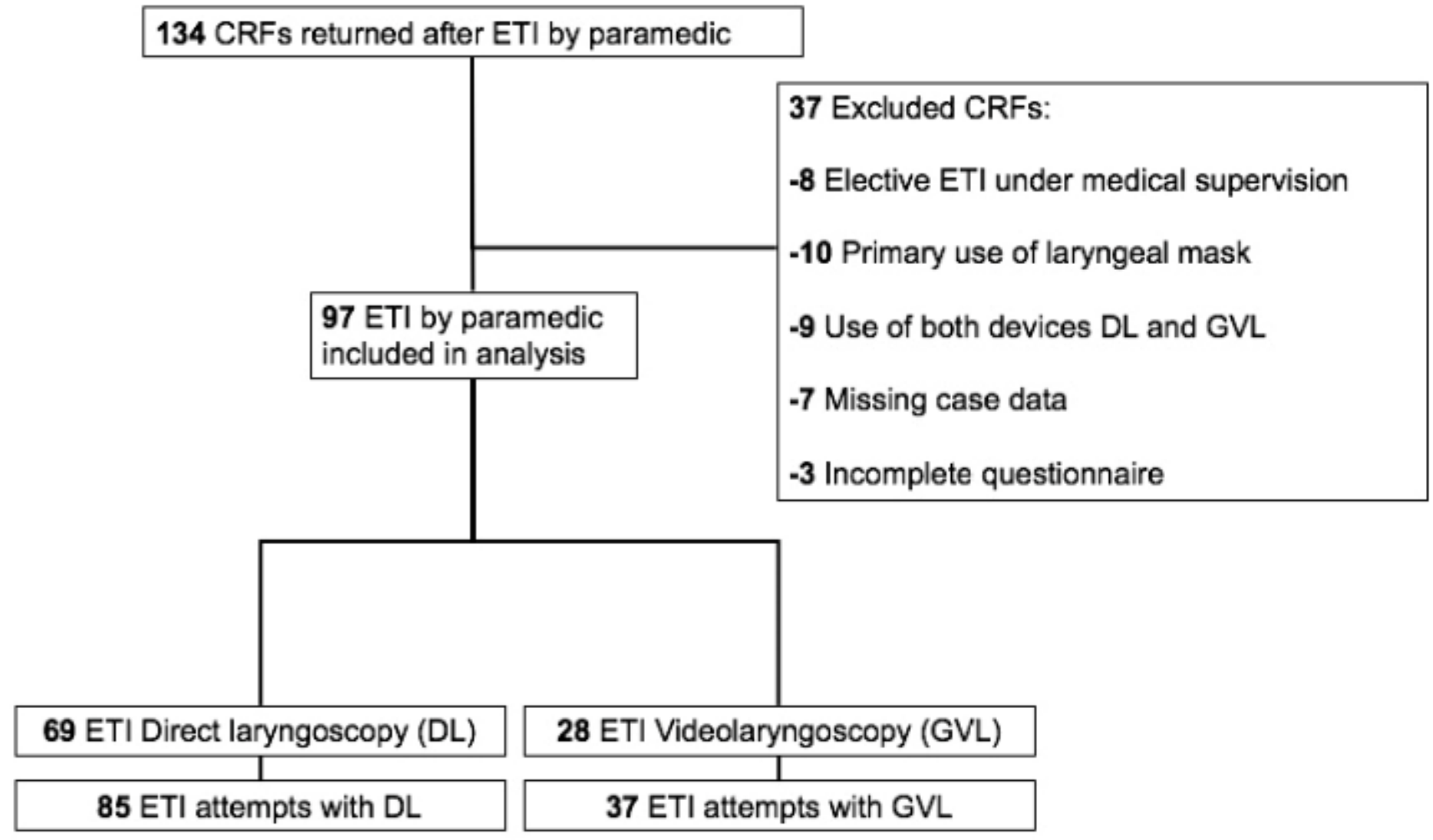

Figure 1

Diagram data evaluation 

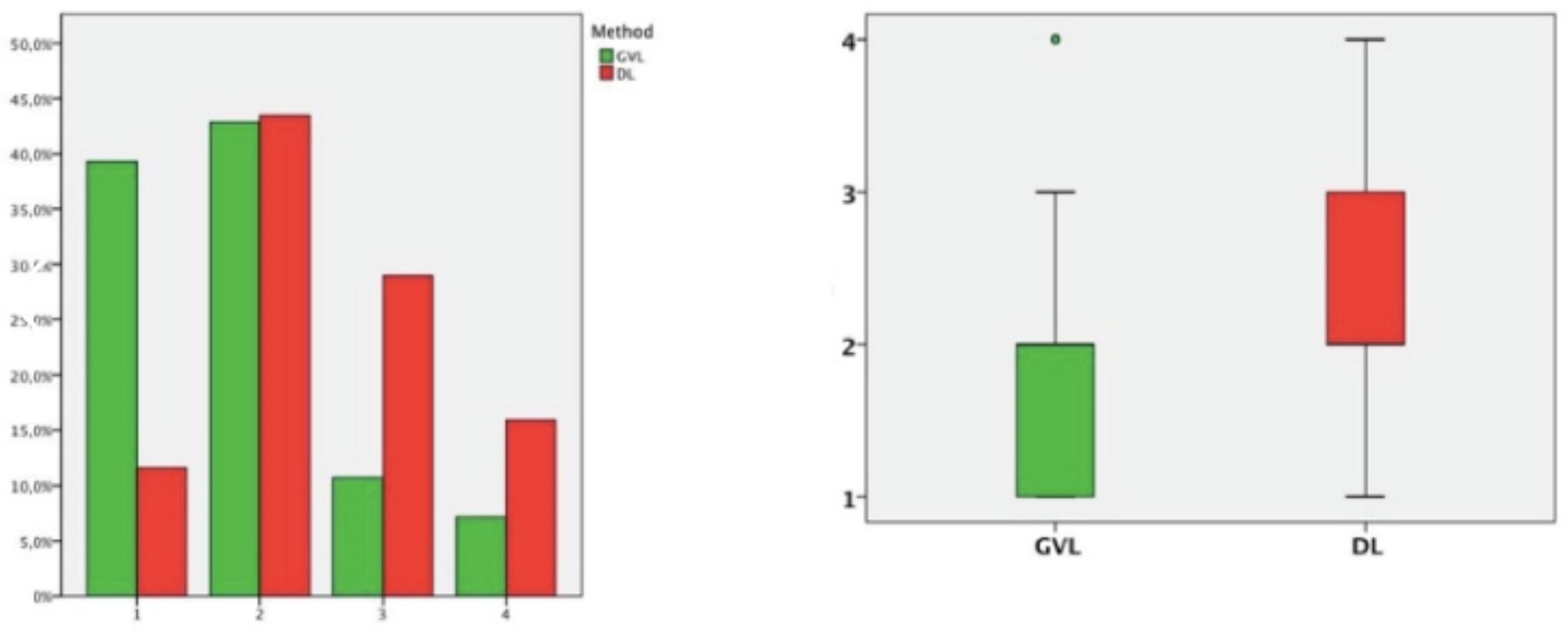

\section{Figure 2}

Frequency distribution of the Cormack and Lehane grading (CL) between videolaryngoscopy (GVL) and direct laryngoscopy (DL) Legend: 1. bar graph x-axis CL Grade, y-axis number in percent, method GVL (green) and DL (red) 2. boxplot x-axis method GVL (green) and DL (red), y-axis CL Grade 1-4

\section{Supplementary Files}

This is a list of supplementary files associated with this preprint. Click to download.

- Table15.docx 